\title{
The Gain and Carrier Density in Semiconductor Lasers under Steady-State and Transient Conditions
}

\author{
Bin Zhao, T. R. Chen, and Amnon Yariv, Fellow, IEEE
}

\begin{abstract}
The carrier distribution functions in a semiconductor crystal in the presence of a strong optical field are obtained. These are used to derive expressions for the gain dependence on the carrier density and on the optical intensitythe gain suppression effect. A general expression for high order nonlinear gain coefficients is obtained. This formalism is then used to describe the carrier and power dynamics in semiconductor lasers above and below threshold in the static and transient regimes.
\end{abstract}

\section{INTRODUCTION}

$\mathrm{T}$ HE statistical properties of electrons and holes in a semiconductor laser active medium are modified by the presence of a strong optical field. The local carrier population are depleted due to stimulated optical transitions thus causing spectral holes in the carrier distribution. These spectral holes in turn tend to be filled by carriers scattering from undepleted portion of the distribution. Incorporation of the above features in semiclassical density-matrix theory using third-order perturbation method in a weak-signal regime [1]-[3] and nonperturbative method in strong-signal regime [4], [5] lead to the description of the so-called gain saturation which has been found to significantly affect the performance of semiconductor lasers [1]-[11]. In the past, quasi-Fermi distributions, which do not account for the spectral hole burning, have been used widely in order to model and calculate various properties in semiconductor laser medium such as recombination current, leakage current, spontaneous emission spectrum, spectral linewidth, and noise [12][16]. However, at high operating powers the deviation of the carrier distributions from the quasi-Fermi distributions can not be neglected in the modeling and calculations.

A linear relation between gain $G$ and injected carrier density $N$ in the active region

$$
G(N)=B_{0}\left(N-N_{0}\right)=G_{0}(N)
$$

Manuscript received March 6, 1991 ; revised August 30, 1992. This work was supported by the Office of Naval Research, the National Science Foundation, and the Army Research Office.

The authors are with T. J. Watson Sr. Laboratories of Applied Physics, California Institute of Technology, Pasadena, CA 91125.

IEEE Log Number 9107765. with $B_{0}$ and $N_{0}$ as material constants, has been used to study the static and transient behavior in double heterostructure (DH) semiconductor lasers because this linear theory is easier to handle theoretically [17]-[20]. However, this linear theory, not accounting for the spectral hole-burning-caused gain saturation, is only valid with no optical field present in the active medium. Expression of the form $G=G_{0} /(1+\epsilon P)$ for the gain has been used to include the gain saturation in studying the dynamic behavior in semiconductor lasers [6], [8], where $P$ is the photon density and $\epsilon$ is a constant. This expression is based on the relation for gain saturation in homogeneously broadened two-level systems and is not proper for semiconductor lasers with electronic band structures since it does not account for spectral hole burning.

In this paper, we derive explicit expressions for the modified carrier distribution functions accounting for the spectral hole burning. These expressions are derived by solving exactly the density-matrix equations and thus are valid in the strong-signal regime. They are important in theoretical modeling and calculations for semiconductor laser medium under high power operation. The error caused by the omission of spectral hole-burning in the distribution functions is calculated. The nonlinear gain terms are obtained from the power-dependent carrier distribution functions and are compared with the previous approach which use a perturbation method to solve the density-matrix equations. We incorporate the nonlinear gain terms in the rate equations to study the gain and carrier density dependence in the steady and transient states of semiconductor lasers. Most of the attractive features of the simple linear theory remain when we include the, more accurate, nonlinear gain terms.

\section{Carrier Distribution, Functions, Gain, and Rate Equations}

In this section we use a semiclassical density-matrix theory to obtain the carrier distribution functions in semiconductor laser medium in the presence of strong optical field. Consider a monochromatic laser field

$$
\mathcal{E}(t)=\frac{1}{2} \varepsilon_{0} e^{-i \omega t}+\text { c.c. }
$$


where $\omega$ is the optical frequency. We use the well-known density-matrix formalism for two-level systems [21], [22] and treat the semiconductor active medium as an ensemble of two-level systems with a rigorous $k$ selection rule applied to the recombining electron-hole pairs. Assuming that electrons in conduction band and holes in valence band have different intraband relaxation time $\tau_{e}$ and $\tau_{h}$, respectively, we rewrite the density-matrix equations in terms of the distribution (occupation) functions for electrons $f_{e}(k)=\rho_{e e}(k)$, and holes $f_{h}(k)=\rho_{h h}(k)$, in the presence of an optical field

$$
\begin{aligned}
\frac{d f_{e}(k)}{d t}= & -i \frac{\mu \varepsilon(t)}{\hbar}\left[\rho_{e h}(k)-\rho_{e h}^{*}(k)\right] \\
& -\frac{f_{e}(k)-f_{e}^{(0)}(k)}{\tau_{e}} \\
\frac{d f_{h}(k)}{d t}= & -i \frac{\mu \varepsilon(t)}{\hbar}\left[\rho_{e h}^{*}(k)-\rho_{e h}(k)\right] \\
& -\frac{f_{h}(k)-f_{h}^{(0)}(k)}{\tau_{h}} \\
\frac{d \rho_{e h}(k)}{d t}= & -i \frac{\mu \varepsilon(t)}{\hbar}\left[f_{e}(k)+f_{h}(k)-1\right] \\
& -i \frac{E_{k}}{\hbar} \rho_{e h}(k)-\frac{\rho_{e h}(k)}{T_{2}}
\end{aligned}
$$

where

$$
f_{e}^{(0)}(k)=\frac{1}{\exp \left(\frac{E_{e}(k)-F_{e}}{k_{B} T}\right)+1}
$$

and

$$
f_{h}^{(0)}(k)=\frac{1}{\exp \left(\frac{E_{h}(k)-F_{h}}{k_{B} T}\right)+1}
$$

are the quasi-Fermi distributions that the electrons and the holes tend to relax to in the presence of the optical field perturbation, $E_{e}(k)$ and $E_{h}(k)$ are the energy of an electron and a hole with wave vector $k$, respectively, $F_{c}$ and $F_{h}$ are the quasi-Fermi energy levels for electrons and holes, respectively, $\rho_{e h}(k)$ is the off-diagonal (electron-hole) density matrix element, $E_{k}$ is the possible transition energy of a electron-hole pair with wave vector $k, T_{2}$ is the interband dephasing time and $\mu$ is the dipole moment matrix element. $f_{e}^{(0)}(k)$ and $f_{h}^{(0)}(k)$ are implicitly dependent on the optical field and injection current in the active region. To make the process self-consistent we use the very different time scales for population relaxation $\left(\tau_{n} \sim 10^{-9} \mathrm{~s}\right)$ and carrier intraband scattering $\left(\tau_{e}, \tau_{h} \sim 10^{-13} \mathrm{~s}\right)$ to solve for carrier density in the presence of injection current and optical power [(24) and (25)]. Equations (3)-(5) can be solved directly for the perturbed distributions $f_{e}(k)$ and $f_{h}(k)$ in a manner similar to that used in a two-level system [22]

$$
\begin{gathered}
f_{e}(k)=f_{e}^{(0)}(k)-\frac{\tau_{e}}{\tau_{e}+\tau_{h}}\left(f_{e}^{(0)}(k)+f_{h}^{(0)}(k)-1\right) \\
\cdot \frac{\mathcal{L}\left(E-E_{k}\right) \frac{P}{P_{s}}}{1+\mathcal{L}\left(E-E_{k}\right) \frac{P}{P_{s}}} \\
f_{h}(k)=f_{h}^{(0)}(k)-\frac{\tau_{h}}{\tau_{e}+\tau_{h}}\left(f_{e}^{(0)}(k)+f_{h}^{(0)}(k)-1\right) \\
\cdot \frac{\mathscr{L}\left(E-E_{k}\right) \frac{P}{P_{s}}}{1+\mathcal{L}\left(E-E_{k}\right) \frac{P}{P_{s}}}
\end{gathered}
$$

where $\mathcal{L}\left(E-E_{k}\right)=E_{T_{2}}^{2} /\left[E_{T_{2}}^{2}+\left(E-E_{k}\right)^{2}\right]$ is the Lorentzian (broadening) function, $E=\hbar \omega$ is the photon energy of the laser field, $E_{T_{2}}=\hbar / T_{2}, P$ is the space average photon density inside the active region, $P_{s}=\hbar^{2} \epsilon_{0} n^{2} / \mu^{2} E$ $\left(\tau_{e}+\tau_{h}\right) T_{2}$ is the saturation photon density and $n$ is the modal refractive index. From (8) and (9) we find that the presence of strong optical field causes spectral holes in the quasi-Fermi-like distributions. $F_{e}$ and $F_{h}$ are related by quasi-neutrality condition

$$
\sum_{k} \frac{1}{V} f_{e}(k)=\sum_{k} \frac{1}{V} f_{h}(k)=N
$$

where $V$ is the volume of the active region.

The solution for $\rho_{e h}(k)$ is

$$
\begin{aligned}
\rho_{e h}(k)= & \frac{\mu \varepsilon_{0}}{2}\left[f_{e}(k)+f_{h}(k)-1\right] \\
& \cdot \frac{1}{\left(E-E_{k}\right)+i E_{T_{2}}} e^{-i \omega t} .
\end{aligned}
$$

The induced polarization can be written quantum mechanically as

$$
\Theta=\operatorname{Tr}[\rho \mu]=\mu \sum_{k} \frac{1}{V}\left[\rho_{e h}(k)+\rho_{e h}^{*}(k)\right]
$$

and classically as

$$
\rho=\operatorname{Re}\left[\epsilon_{o} \chi \mathcal{E}_{0} e^{-i \omega t}\right]
$$

where $\chi$ is the macroscopic susceptibility. Equations (11)(13) lead to an expression for the exponential gain coefficient at photon energy $E$

$$
G(E)=A \sum_{k} \frac{1}{V}\left(f_{e}(k)+f_{h}(k)-1\right) \mathcal{L}\left(E-E_{k}\right)
$$

where $A=E \mu^{2} / \hbar c \epsilon_{o} n E_{T_{2}}$. Using (8), (9), (14) and assuming $P / P_{s}<1$, the gain can be expressed as

$$
\begin{aligned}
G(E)= & G_{0}(E)-G_{1}(E) \frac{P}{P_{s}} \\
& +\cdots+(-1)^{n} G_{n}(E)\left(\frac{P}{P_{s}}\right)^{n}+\cdots
\end{aligned}
$$


where the linear gain coefficient $G_{0}(E)$, first order nonlinear gain coefficient $G_{1}(E)$ and higher order nonlinear gain coefficients $G_{n}(E)(n>1)$ are given by

$$
\begin{aligned}
& G_{0}(E)=A \sum_{k} \frac{1}{V}\left(f_{e}^{0}(k)+f_{h}^{0}(k)-1\right) \mathcal{L}\left(E-E_{k}\right) \\
& G_{1}(E)=A \sum_{k} \frac{1}{V}\left(f_{e}^{0}(k)+f_{h}^{0}(k)-1\right) \mathcal{L}^{2}\left(E-E_{k}\right) \\
& G_{n}(E)=A \sum_{k} \frac{1}{V}\left(f_{e}^{0}(k)+f_{h}^{0}(k)-1\right) \mathcal{L}^{n+1}\left(E-E_{k}\right)
\end{aligned}
$$

$$
(n>1) \text {. }
$$

Comparing with [1]-[3], a general expression for high order nonlinear gain coefficients is given here. In the commonly used third-order perturbation theory [1]-[3], the density-matrix equations are solved by perturbation method. In our approach, the density-matrix equations are solved exactly. This facilitates the task of obtaining expression for the high order nonlinear gain coefficients.

In the analysis which follows we consider the first two terms in (15), so it is valid for $P / P_{s} \ll 1$. We employ (16) and (17) to calculate the linear gain spectrum $G_{0}(E)$ and the nonlinear gain spectrum $G_{1}(E)$ for DH InGaAsP/ InP lasers with $\lambda \approx 1.3 \mu \mathrm{m} . m_{e}^{*}=0.056 m_{0}, m_{h}^{*}=$ $0.69 m_{0}, n=3.5, T_{2}=0.1 \mathrm{ps}, \mu=7.7 \times 10^{-29} \mathrm{C} \cdot \mathrm{m}$ were used in the calculation, where $m_{e}^{*}, m_{h}^{*}$, and $m_{0}$ are effective mass of electrons, effective mass of holes, and free-electron mass, respectively. The quasi-Fermi distributions and injected carrier density are related by the quasi-neutrality condition

$$
N=\sum_{k} \frac{1}{V} f_{e}^{0}(k)=\sum_{k} \frac{1}{V} f_{h}^{0}(k) .
$$

Using the unperturbed quasi-Fermi distributions to calculate the carrier density [(19)] is an approximation. The derived solutions for the field-perturbed carrier distribution functions allow us to determine quantitatively the errors involved. Using (8) and (9), the errors in the injected electron density $\left(\delta N_{e}\right)$ and hole density $\left(\delta N_{h}\right)$ can be evaluated by

$$
\begin{aligned}
\delta N_{e}= & \sum_{k} \frac{1}{V}\left(f_{e}^{0}(k)-f_{e}(k)\right) \\
= & \sum_{k} \frac{1}{V} \frac{\tau_{e}}{\tau_{e}+\tau_{h}}\left(f_{e}^{(0)}(k)+f_{h}^{(0)}(k)-1\right) \\
& \cdot \frac{\mathscr{L}\left(E-E_{k}\right) \frac{P}{P_{s}}}{1+\mathscr{L}\left(E-E_{k}\right) \frac{P}{P_{s}}}
\end{aligned}
$$

$$
\begin{aligned}
\delta N_{h}= & \sum_{k} \frac{1}{V}\left(f_{h}^{0}(k)-f_{h}(k)\right) \\
= & \sum_{k} \frac{1}{V} \frac{\tau_{h}}{\tau_{e}+\tau_{h}}\left(f_{e}^{(0)}(k)+f_{h}^{(0)}(k)-1\right) \\
& \cdot \frac{\mathcal{L}\left(E-E_{k}\right) \frac{P}{P_{s}}}{1+\mathscr{L}\left(E-E_{k}\right) \frac{P}{P_{s}}} .
\end{aligned}
$$

$\tau_{e}=0.16 \mathrm{ps}$ and $\tau_{h}=0.04 \mathrm{ps}$ were estimated from the experimentally determined mobilities for electrons and holes [23], [24]. The relative carrier density errors $\delta N / N$ and $\delta N_{h} / N$ are less than $0.25 \%$ for $N<5 \times 10^{19} \mathrm{~cm}^{-3}$ and $P / P_{s}<0.2$. This spectral hole-burning-caused error increases more or less linearly as $N$ or $P / P_{s}$ increases. The error is smaller for holes since the holes have a shorter intraband relaxation time.

Fig. 1 shows the peak linear gain coefficient $G_{0}^{m}$ versus injected carrier density $N$ in the active layer obtained from the theoretical $G_{0}(E)$ curves. The corresponding nonlinear gain coefficient $G_{1}^{m}$ is also shown in Fig. 1. We notice that the photon energies at which $G_{0}(E)$ and $G_{1}(E)$ peak are nearly the same. The lasing threshold is determined mostly by the linear gain. From Fig. 1, the dependence of $G_{0}^{m}$ and $G_{1}^{m}$ on carrier density $N$ above transparency can be approximated closely by

$$
\begin{aligned}
& G_{0}^{m}(N)=B_{0}\left(N-N_{0}\right) \\
& G_{1}^{m}(N)=B_{1}\left(N-N_{1}\right)
\end{aligned}
$$

with $B_{0}=2.8 \times 10^{-16} \mathrm{~cm}^{2}, N_{0}=2.4 \times 10^{18} \mathrm{~cm}^{-3}$, $B_{1}=1.4 \times 10^{-16} \mathrm{~cm}^{2}$, and $N_{1}=1.6 \times 10^{18} \mathrm{~cm}^{-3}$. These results are consistent with those which were obtained using perturbation method to solve the density-matrix equations [1]-[3].

The gain coefficients including the gain suppression term $G_{1}^{m}$ are incorporated in the single-mode rate equations for the photon density and the carrier density

$$
\begin{aligned}
& \frac{d N}{d t}=\frac{J}{e d}-\frac{N}{\tau_{n}}-G_{0}^{m} v_{g} P+G_{1}^{m} v_{g} \frac{P^{2}}{P_{s}} \\
& \frac{d P}{d t}=\Gamma G_{0}^{m} v_{g} P-\Gamma G_{1}^{m} v_{g} \frac{P^{2}}{P_{s}}-\frac{P}{\tau_{p}}+\Gamma \theta \frac{N}{\tau_{n}}
\end{aligned}
$$

where $J$ is the radiative injection current density, $\tau_{n}$ is the carrier recombination lifetime, $\tau_{p}=\left(v_{g} \alpha_{t}\right)^{-1}=\left[v_{g}\left(\alpha_{i}+\right.\right.$ $\left.\left.1 / 2 L \ln 1 / R_{1} R_{2}\right)\right]^{-1}$ is the photon life time, $v_{g}$ is the photon group velocity, $\alpha_{i}$ is the internal loss constant, $\theta$ is the spontaneous emission coupling factor defined as the ratio of the rate of spontaneous emission into the lasing mode to the total spontaneous emission rate, $\Gamma$ is the optical confinement factor, and $d$ is the active layer thickness.

\section{Results And Discussion}

The gain, photon density, and injection current density in steady-state operation are obtained directly from (24) 


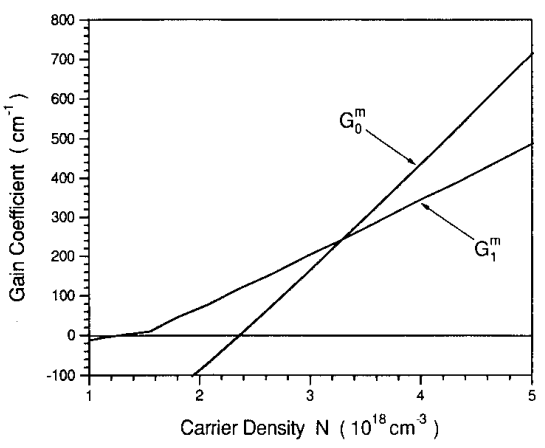

Fig. 1. Peak linear gain coefficient $G_{0}^{m}$ and corresponding first order nonlinear gain coefficient $G_{1}^{m}$ as a function of injected carrier density $N$.

and (25)

$$
\begin{gathered}
G(N)=\frac{\left(G_{0}^{m}+\frac{\alpha_{t}}{\Gamma}\right)-\sqrt{\left(G_{0}^{m}-\frac{\alpha_{t}}{\Gamma}\right)^{2}+4 \theta \frac{N}{\tau_{n}} \frac{G_{1}^{m}}{v_{g} P_{s}}}}{2} \\
P=\frac{\left(G_{0}^{m}-\frac{\alpha_{t}}{\Gamma}\right)+\sqrt{\left(G_{0}^{m}-\frac{\alpha_{t}}{\Gamma}\right)+4 \theta \frac{N}{\tau_{n} \frac{G_{1}^{m}}{v_{g} P_{s}}}} P_{s}}{2 G_{1}^{m}}
\end{gathered}
$$

$$
\begin{aligned}
J & =e d\left(\frac{N}{\tau_{n}}+G_{0}^{m} v_{g} P-G_{1}^{m} v_{g} \frac{P^{2}}{P_{s}}\right) \\
& =e d(1-\theta) \frac{N}{\tau_{n}}+\frac{e d}{\tau_{p} \Gamma} P .
\end{aligned}
$$

To investigate $G, N$, and $P$ we use $P_{s}=6.7 \times 10^{16} \mathrm{~cm}^{-3}$ estimated using the relevant parameters given above, $\tau_{n}=3 \times 10^{-9} \mathrm{~s}, \Gamma=0.35$, and $\alpha_{i}=20 \mathrm{~cm}^{-1}$ for a InGaAsP-InP laser with mirror reflectivities $R_{1}=R_{2}=$ 0.3 and cavity length $L=300 \mu \mathrm{m} . P / P_{s}$ was estimated to be less than 0.2 for a InGaAsP-InP laser without facet coating up to the highest power $50 \mathrm{~mW}$ per facet [25]. For lasers with even higher output power (15), (26)-(28) are not valid and one has to employ strong-signal theory to more accurately include the effect of gain saturation.

In Fig. 2 we show the computed gain and carrier density relation for several different $\theta$ values above transparency. The gain has a linear dependence on the carrier density below lasing threshold and saturates abruptly to $\alpha_{t} / \Gamma$ above lasing threshold. This hard thresholding is softened when we include the effect of spontaneous emission as evident from Fig. 2 for $\theta \neq 0$. Omitting the spontaneous emission coupling, i.e., $\theta=0$, the gain and the photon density can be written as

$$
G(N)=\frac{\left(G_{0}^{m}+\frac{\alpha_{t}}{\Gamma}\right)-\left|G_{0}^{m}-\frac{\alpha_{t}}{\Gamma}\right|}{2}
$$

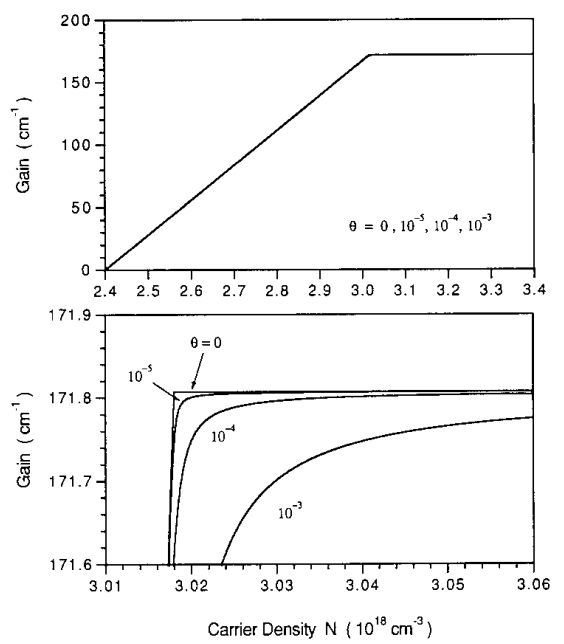

Fig. 2. Gain as a function of injected carrier density, for various values of spontaneous emission coupling factor $\theta$.

$$
P=\frac{\left(G_{0}^{m}-\frac{\alpha_{t}}{\Gamma}\right)+\left|G_{0}^{m}-\frac{\alpha_{t}}{\Gamma}\right|}{2 G_{1}^{m}} P_{s} .
$$

Below threshold, $\Gamma G_{0}^{m}<\alpha_{t}$, the gain and photon density are given by

$$
\begin{aligned}
G(N) & =G_{0}^{m}(N)=B_{0}\left(N-N_{0}\right) \\
P & =0
\end{aligned}
$$

and $G$ depends on $N$ linearly. Above threshold $\Gamma G_{0}^{m}>$ $\alpha_{t}$, the gain and photon density are given by

$$
\begin{aligned}
G(N) & =\frac{\alpha_{t}}{\Gamma} \\
P & =\frac{\left(G_{0}^{m}-\frac{\alpha_{t}}{\Gamma}\right)}{2 G_{1}^{m}} P_{s}
\end{aligned}
$$

the gain is clamped. These results indicate the threshold is determined by the linear gain constant $G_{0}^{m}$. And in describing the steady-state operation of semiconductor lasers, the nonlinear gain coefficient $G_{1}^{m}$ is as important as in describing the dynamic behavior of a semiconductor laser. It is interesting to compare the above results with those of the linear theory when the gain dependence on carrier density is linear [(1)] even above lasing threshold. The corresponding expressions can be easily obtained by solving the rate equations (24) and (25) with $G_{1}^{m}=0$. The photon density in the linear theory is

$$
P=\frac{\Gamma \theta N / \tau_{n}}{v_{g}\left(\alpha_{t}-\Gamma G_{0}^{m}\right)} .
$$

The $P-N$ relation (35) describes the semiconductor lasers as pure narrow-band spontaneous emission amplifiers while the $P-N$ relation (27) indicates that the semicon- 

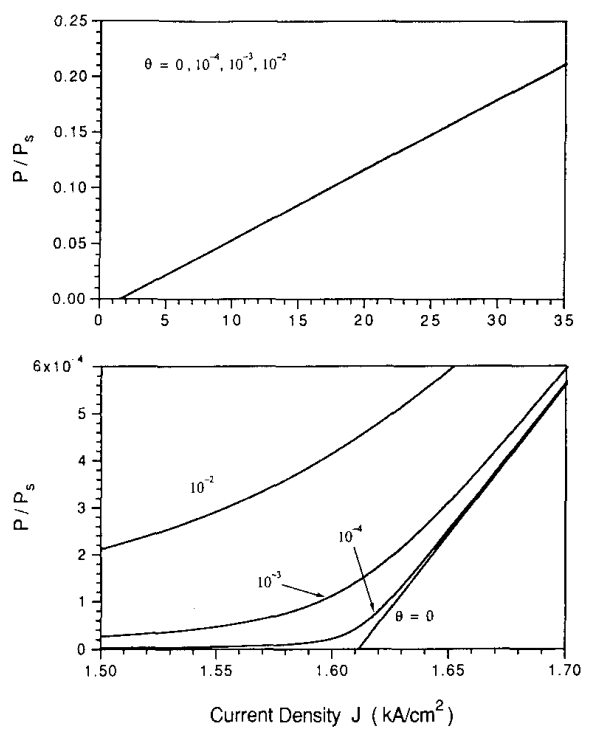

Fig. 3. Normalized photon density in active region $P / P_{s}$ as a function of injection current density $J$, for $\theta=0,10^{-4}, 10^{-3}, 10^{-2}$.

ductor lasers are oscillators only partially driven by the spontaneous emission. In the nonlinear theory, $G_{0}^{m}(N)>$ $\alpha_{t} / \Gamma$ above threshold since the total gain is clamped but not the linear gain. In the linear theory, the linear gain is the total gain, so $G_{0}^{m}(N)<\alpha_{t} / \Gamma$ above threshold.

Fig. 3 shows the computed photon density, which is proportional to the optical output power, as a function of the injection current density. Well above threshold, the photon density and the injection current density have a nearly linear relation. From (28), we see that this nearly linear relation is due to the small variation of injected carrier density above threshold. In Fig. 3, we also show how the spontaneous emission coupling factor $\theta$ affects the optical power versus injection current characteristics near threshold for semiconductor lasers. For larger $\theta$, the lasing threshold is not well defined.

The computed $N-J$ curves in Fig. 4 show that although the slopes of the $N-J$ curves change dramatically as threshold is crossed, the injected carrier density in the active region, unlike the total gain, does not clamp above threshold. As a direct consequence, the quasi-Fermi levels of electrons in conduction band and holes in valence band are not clamped in the lasing regime. The nonclamping of injected carrier density under oscillation may contribute to the increase of leakage current above threshold which is observed experimentally [26] in DH lasers. This will also lead to the nonclamping of various nonradiative recombination mechanisms, such as the Auger recombination, which depend on the carrier density.

In our analysis, we have taken the internal quantum efficiency to be unity and did not include the various nonradiative recombination processes and carrier leakage processes in the laser devices to get the total injection current density. Their influence can be easily incorporated to
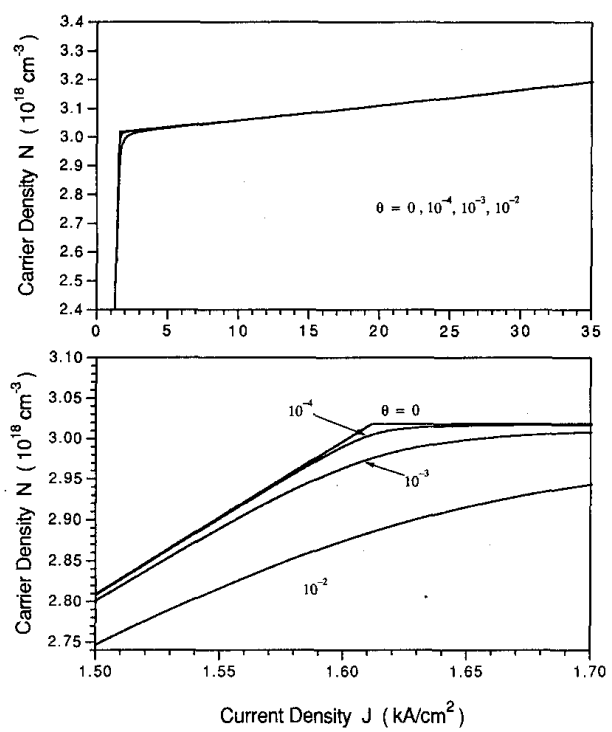

Fig. 4. Injected carrier density within the active region $N$ as a function of injection current density $J$, for $\theta=0,10^{-4}, 10^{-3}, 10^{-2}$.

modify the injection current density if the thermal effect is not considered.

This analysis is also valid for other types of semiconductor lasers, such as quantum-well (QW) lasers. The gain and carrier density dependence expression (26) is still valid for QW lasers but $G_{0}^{m}$ and $G_{1}^{m}$ will have different dependence on $N$. One consequence of the nonclamping of quasi-Fermi levels under oscillation in QW lasers is the observed injection-current controlled lasing wavelength switching [27].

We employed the time-dependent rate equations (24) and (25) to study the gain and carrier density dependence in the transient regime. The rate equations were solved numerically by assuming a constant injection current switched on at $t=0$. In Fig. 5, we show the gain and carrier density $(G-N)$ dependence using our nonlinear gain (NLG) theory during the transient at two injection levels, $J=1.2 J_{t h}$ and $J=12 J_{t h}$, where $J_{t h}=1.61 \mathrm{kA} / \mathrm{cm}^{2}$ was obtained from Fig. 3 and $\theta=10^{-4}$ was used. It is shown that the gain and carrier density has a linear relation at the beginning of the transient and a nonlinear damped oscillation behavior about the steady-state point. At low injection level, the trace of the oscillation cycles is ellipse-like with a large value of eccentricity. The nonlinearity of the $G-N$ dependence is enhanced at the high injection levels. The $G-N$ dependence shows larger maximum oscillation amplitudes and fewer oscillation cycles to reach the steady-state point at the high injection level. Figs. 6 and 7 show the time evolution of the carrier density, the photon density, and the total gain at the two different injection levels. For comparison, we also show the evolution of the corresponding variables given by the linear gain (LG) theory. The damping rate of relaxation oscillation in the NLG theory is larger than that in the LG theory. 

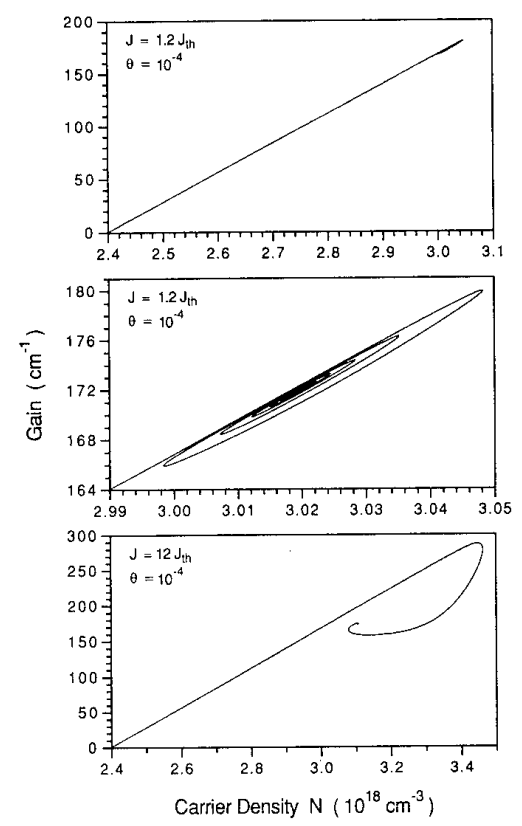

Fig. 5. Gain and carrier density dependence in transient process at different injection levels, $J=1.2 J_{t h}$ and $J=12 J_{t h}, \theta=10^{-4}$.
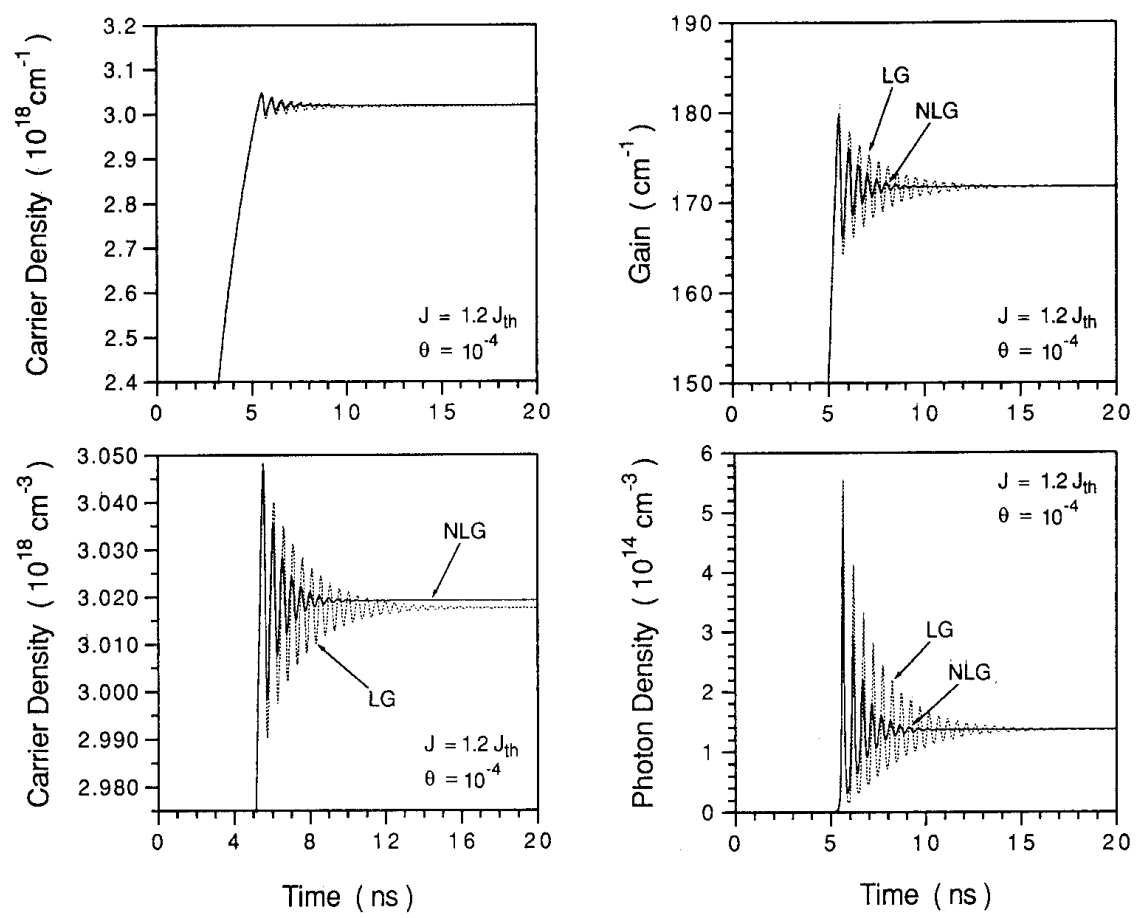

Fig. 6. Time evolution of carrier density, photon density, and gain at low current injection $J=1.2 J_{d b}, \theta=10^{-4}$.

And amplitude of relaxation oscillation in the NLG theory is smaller than that in the LG theory. These differences are larger at the high injection level. In Figs. 6 and 7, we find that the steady-state carrier density in the NLG theory
$\left(N_{N L G}\right)$ is larger than that in the LG theory $\left(N_{L G}\right)$ under the same injection condition. The reason for $N_{N L G}>N_{L G}$ is that $G_{0}^{m}(N)>\alpha_{t} / \Gamma$ in the NLG theory and $G_{0}^{m}(N)<$ $\alpha_{t} / \Gamma$ in the LG theory. 

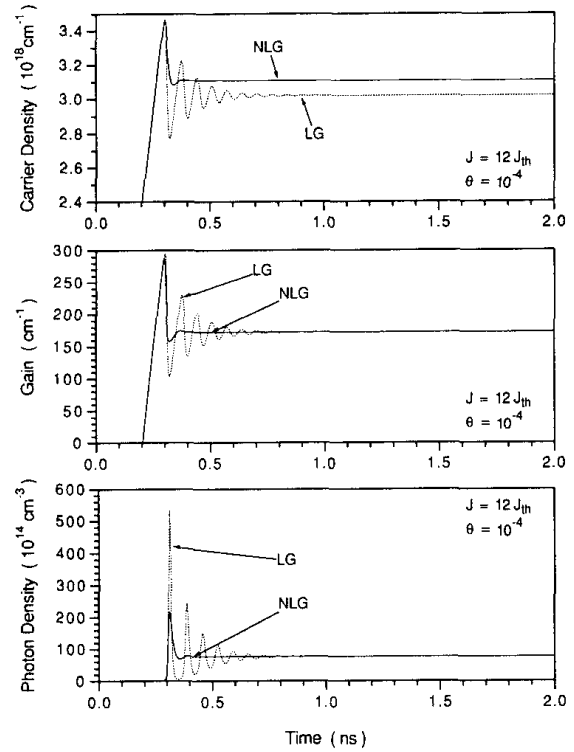

Fig. 7. Time evolution of carrier density, photon density, and gain at high current injection $J=12 J_{t h}, \theta=10^{-4}$.

The analysis presented above assumes a single-mode operation. This is often the case with $\mathrm{DH}$ [17] and QW [28] lasers over wide injection current range above threshold in steady-state operation. In the transient process, the lasers are usually in multimode operation at the beginning [18], [19]. The multimode operation will cause some modification to our analysis, but the basic features will remain the same.

\section{CONCLUSION}

Expressions for the carrier distribution functions in semiconductor laser medium in the presence of strong optical field are given. From this we obtained expressions for the dependence of the gain on carrier density and on the optical intensity which includes the effect of optical field gain saturation. A general expression for high order nonlinear gain coefficients has been obtained. We have considered the optical power-dependent deviation from linearity of the gain-carrier density characteristic of semiconductor lasers. One single explicit expression is given to describe the gain-carrier density dependence both below and above lasing threshold in steady-state operation. The derived explicit expression for the photon density within the active region shows the importance of nonlinear gain in describing the behavior of semiconductor lasers at steady-state and it also shows that semiconductor lasers are oscillators partially driven by the spontaneous emission. The influence of spontaneous emission coupling into the lasing mode on the optical power vs. injection current characteristics is manifest in this nonlinear theory. In the transient regime, the nonlinearity of the gain and carrier density dependence is illustrated at different injection levels and the gain-carrier density dependence shows a large nonlinearity at high injection levels.

\section{REFERENCES}

[1] M. Yamada and Y. Suematsu, "Analysis of gain suppression in undoped injection lasers," J. Appl. Phys., vol. 52, pp. 2653-2664, 1981.

[2] M. Yamada, "Transverse and longitudinal mode control in semiconductor injection lasers," IEEE J. Quantum Electron., vol. QE-21, pp. $1365-1380,1983$.

[3] M. Asada and Y. Suematsu, "Density-matrix theory of semiconductor lasers with relaxation broadening model-Gain and gain-suppression in semiconductor lasers,"' IEEE J. Quantum Electron., vol. QE21, pp. 434-442, 1985

[4] W. W. Chow, G. C. Dente, and D. Depatie, "Saturation effect in semiconductor lasers," IEEE J. Quantum Electron., vol. QE-23, pp. 1314-1320, 1987.

[5] G. P. Agrawal, "Spectral hole-burning and gain saturation in semiconductor lasers: Strong-signal theory,"'J. Appl. Phys., vol. 63, pp. 1232-1235, 1988.

[6] D. P. Channin, "Effect of gain saturation on injection laser switching,"'J. Appl. Phys., vol. 50, pp. 3858-3860, 1979.

[7] R. S. Tucker, "High-speed modulation of semiconductor lasers," $J$ Lightwave Technol., vol. LT-3, pp. 1180-1192, 1985.

[8] J. E. Bowers, B. Roe Hemenway, A. H. Gnauck, and D. P. Wilt, "High-speed InGaAsP constricted-mesa lasers," IEEE J. Quantum Electron., vol. QE-22, pp. 833-844, 1986.

[9] C. B. Su and V. A. Lanzisera, "Ultra-high-speed modulation of $1.3-$ $\mu \mathrm{m}$ InGaAsP diode lasers," IEEE J. Quantum Electron., vol. QE22 , pp. $1568-1578,1986$.

[10] G. P. Agrawal, "Intensity dependence of the linewidth enhancement factor and its implications for semiconductor lasers," IEEE Photon. Technol. Lett., vol, 1, pp. 212-214, 1989.

[11] G. Morthier, P. Vankwikelberge, F, Buytaert, and R. Bates, "Influence of gain nonlinearities on the linewidth enhancement factor in semiconductor lasers,"' IEE Proc., vol. 137, pt. J, pp. 30-32, 1990.

[12] M. Asada, A. R. Adams, K. E. Stubkjaer, Y. Suematsu, Y. Itaya, and $S$. Arai, "The temperature dependence of the threshold current of GaInAsP/InP DH lasers," IEEE J. Quantum Electron., vol. QE17, pp. 611-619, 1981.

[13] K. Vahala, L. C. Chiu, S. Margalit, and A. Yariv, "On the linewidth enhancement factor $\alpha$ in semiconductor injection lasers," Appl. Phys. Lett., vol. 42, pp. 631-633, 1983.

[14] K. Vahala and A. Yariv, "Occupation fluctuation noise: a fundamental source of linewidth broadening in semiconductor lasers," Appl. Phys. Lett., vol. 43, pp. 140-142, 1983.

[15] Y. Arakawa and A. Yariv, "Quantum well lasers-Gain, spectra, dynamics," IEEE J. Quantum Electron., vol. QE-22, pp. 1887-1899, 1986.

[16] S. R. Chinn, P. S. Zory, and A. R. Reisinger, "A model for GRINSCH-SQW diode lasers," IEEE J. Quantum Electron., vol. 24, pp. 2191-2214, 1988.

[17] T. P. Lee, C. A. Burrus, J. A. Copeland, A. G. Dentai, and D. Marcuse, "Short-cavity InGaAsP injection lasers: Dependence of mode spectra and single-longitudinal-mode power on cavity length," IEEE J. Quantum Electron., vol. QE-18, pp. 1101-1113, 1982.

[18] K. Otsuka and S. Tarucha, "Theoretical studies on injection locking and injection induced modulation of laser diodes," IEEE J. Quantum Electron., vol. QE-17, pp. 1515-1521, 1981.

[19] D. Marcuse and T. P. Lee, "On approximate analytical solutions of rate equations for studying transient spectra of injection lasers," IEEE J. Quantum Electron., vol. QE-19, pp. 1397-1406, 1983.

[20] J. E. Carroll, Rate Equations in Semiconductor Electronics. Cambridge: Cambridge Univ. Press, 1985, ch. 6, 7.

[21] M. Sargent III, M. O. Scully, and W. E. Lamb, Jr., Laser Physics. Reading, MA: Addison-Wesley, 1974, ch. 7.

[22] A. Yariv, Quantum Electronics, 3rd ed. New York: Wiley, 1989, ch. 8 .

[23] P. D. Greene, S. A. Wheeler, A. R. Adams, A. N. El-Sabbahy, and C. N. Ahmad, "Background carrier concentration and electron mobility in LPE $\operatorname{In}_{1-x} \mathrm{Ga}_{x} \mathrm{As}_{y} \mathrm{P}_{1-y}$ layers,' Appl. Phys. Lett., vol. 35, pp. 78-80, 1979 .

[24] J. R. Hayes, A. R. Adams, and P. R. Greene, "Mobility of holes in the quaternary alloy $\mathrm{In}_{1-x} \mathrm{Ga}_{x} \mathrm{AS}_{y} \mathrm{P}_{1-y}$," Electron. Lett., vol. 16 , pp. 283-284, 1980.

[25] T. R. Chen, B. Zhao, Y. H. Zhuang, and A. Yariv, "Combined high power and high frequency operation of InGaAsP/InP lasers at 1.3 $\mu \mathrm{m}$," Fiber and Integrated Optics, vol. 9, pp. 347-366, 1990.

[26] T. R. Chen, S. Margalit, U. Koren, K. L. Yu, L. C. Chiu, A. Has- 
son, and A. Yariv, "Direct measurement of the carrier leakage in an InGaAsP/InP laser," Appl. Phys. Lett., vol, 42, pp. 1000-1002, 1983.

[27] Y. Tokuda, N. Tsukada, K. Fujiwara, K. Hamanaka, and T. Nakayama, "Widely separate wavelength switching of single quantum well laser diode by injection-current control,"'Appl. Phys. Lett., vol. 49, pp. $1629-1631,1986$

[28] B. Zhao, T. R. Chen, and A. Yariv, unpublished.

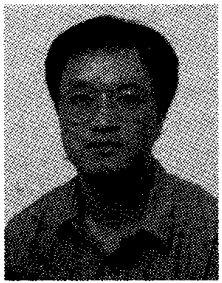

Bin Zhao was born in Beijing, China, in 1963. He received the B.S. degree in electronics engineering from Tsinghua University, Beijing, China, in 1985, and the M.S. degree in applied physics from the California Institute of Technology, Pasadena, in 1988.

Currently, he is pursuing the Ph.D. degree at the California Institute of Technology and his research interests are in the area of semiconductor optoelectronic devices.

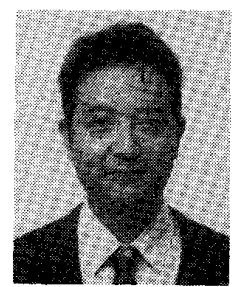

T. R. Chen was born in China on December 4 1939. He received a degree in physics from Wuhan University in 1962.

He then joined the faculty of the University of Electronic Science and Technology in China, becoming a full professor in 1984. He was appointed a visiting professor at the University of Illinois. Urbana-Champaign, for the summer of 1981 , and was appointed a visiting associate in the Department of Applied Physics, California Institute of Technology, Pasadena, from 1980 to 1983 and again from 1985 until the present. His research interests have been in the area of optical resonator theory, optical communication. semiconductor photonic devices, and optoelectronic integration. He is an author or coauthor of three books (in Chinese) and approximately 60 professional papers.

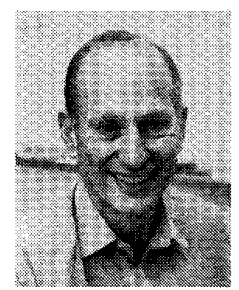

Amnon Yariv (S'56-M'59-F'70) was born in Israel and obtained the B.S., M.S., and Ph.D. degrees in electrical engineering from the University of California, Berkeley, in 1954, 1956, and 1958 . respectively.

He joined Bell Telephone Laboratories, Murray $\mathrm{Hill}, \mathrm{NJ}$, in 1959 , at the early stages of the laser effort. He joined the California Institute of Technology, Pasadena, in 1964 as an Associate Professor of Electrical Engineering, and became a Professor in 1966. In 1980 he was the Thomas G

Myers Professor of Electrical Engineering and Applied Physics. On the technical side, he took part (with various co-workers) in the discovery of a number of early solid-state laser systems, in proposing and demonstrating semiconductor based integrated optics technology, and in pioneering the field of phase conjugate optics. His present research efforts are in the areas of nonlinear optics, semiconductor lasers, and integrated optics. He has published widely in the laser and optics fields some 300 papers and has written a number of basic texts in quantum electronics, optics, and quantum mechanics. He is also a founder and chairman-of-the-board of the Ortel Corp., Alhambra, CA, and of Accuwave Corp.

Dr. Yariv is a member of the American Physical Society, Phi Beta Kappa. the American Academy of Arts and Sciences, and the National Academy of Engineering, and a Fellow of the Optical Society of America. He received the 1980 Quantum Electronics Award of the IEEE, the 1985 Uni versity of Pennsylvania Pender Award, and the 1986 Optical Society of America Ives Medal. 